\title{
OPEN A miniaturized spectrometer for NMR relaxometry under extreme conditions
}

Received: 16 June 2019

Accepted: 22 July 2019

Published online: 01 August 2019
Yiqiao Tang, David McCowan \& Yi-Qiao Song

With the advent of integrated electronics, microfabrication and novel chemistry, NMR (Nuclear Magnetic Resonance) methods, embodied in miniaturized spectrometers, have found profound uses in recent years that are beyond their conventional niche. In this work, we extend NMR relaxometry on a minute sample below $20 \mu \mathrm{L}$ to challenging environment of $150^{\circ} \mathrm{C}$ in temperature and 900 bar in pressure. Combined with a single-board NMR spectrometer, we further demonstrate multidimensional NMR relaxometries capable of resolving compositions of complex fluids. The confluence of HTHP (high-pressure high-temperature) capability, minimal sample volume, and reduced sensor envelop and power budget creates a new class of mobile NMR platforms, bringing the powerful analytical toolkit in a miniaturized footprint to extreme operating conditions.

NMR, considered as one of the most potent analytical methods, traditionally demands superconducting magnets ${ }^{1}$, sizable electronics, and intricate probe and antenna placements ${ }^{2,3}$. Only recently, owing to the advancement in permanent-magnet design ${ }^{4,5}$, electronics integration ${ }^{6,7}$, and antenna miniaturization ${ }^{8,9}$, portable NMR systems ${ }^{10}$ have emerged as a viable surrogate. Thanks to the reductions in footprint, maintenance needs and price tag, the miniaturized sensor assemblies have extended their uses beyond conventional NMR laboratories to a broad range of "field" applications, including point-of-care medical diagnostics ${ }^{11,12}$, flow metering ${ }^{13,14}$, fluid authentication $^{15,16}$, porous material characterization ${ }^{17,18}$, and artefact preservation ${ }^{19,20}$.

Constrained by the current design of permanent magnet, many miniaturized systems focus on measuring NMR relaxations ${ }^{11,21,22}$ that originate from interactions of the spin system with its molecular environment ${ }^{23}$. A major deficiency of these small systems, however, is their limit on operating environment to ambient conditions. While applications of NMR abound at elevated pressure and/or temperature settings, such as in subsurface explorations ${ }^{24}$, polymer dynamics ${ }^{25,26}$, catalysis ${ }^{27}$, hydrogen storage ${ }^{28}$, and gas adsorption in nanoporous materials ${ }^{29,30}$, those measurements always demand complex mechanical designs, large space and numerous pieces of equipment. In this context, miniaturized and integrated NMR platforms with HTHP (high-temperature, high-pressure) capability could further the technique to a substantially broadened usage.

A number of challenges arise when conduct NMR relaxometry within a minimal envelop under HTHP conditions. For example, the reduced volume leads to degraded SNR (signal-to-noise ratio), which further deteriorates at high temperatures. The small volume also corresponds to an increased surface-to-volume ratio of the sample contained in a capillary; accordingly, surface relaxations from the walls of sample tubes, incurred by paramagnetic impurities ${ }^{31}$ and geometrical defects ${ }^{32,33}$, need to be meticulously characterized and rectified. At elevated temperatures, sample relaxation times tend to increase owing to the accelerated molecular motion, demanding systems of superior stability that maintain phase coherence through an extended experimental duration. Finally, the HTHP requirements put further constraints on the selection of capillary materials, the probe design, and associated electronics.

We hereby report the development of a miniaturized NMR platform that is capable of carrying out high-quality relaxation measurements (both $T_{1}$ and $T_{2}$ ) within a micro-autoclave up to $150^{\circ} \mathrm{C}$ and 900 bar hydraulic pressure. In particular, $T_{2} \sim 10 \mathrm{~s}$ is obtained from a pressurized water sample at $150^{\circ} \mathrm{C}$ and $137 \mathrm{bar}$, with a detection volume below $20 \mu \mathrm{L}$. We further show an integrated system, based on an NMR-ASIC (Application Specific Integrated Circuitry) chipset $^{6}$ and a pressure-compensated NMR probe, that can perform a wide range of NMR measurements on a rich diversity of complex fluids. 
(A)

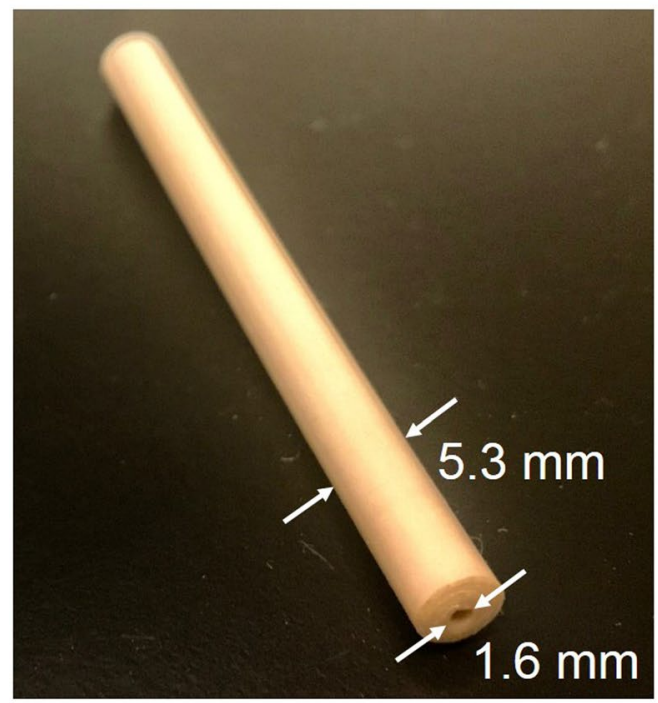

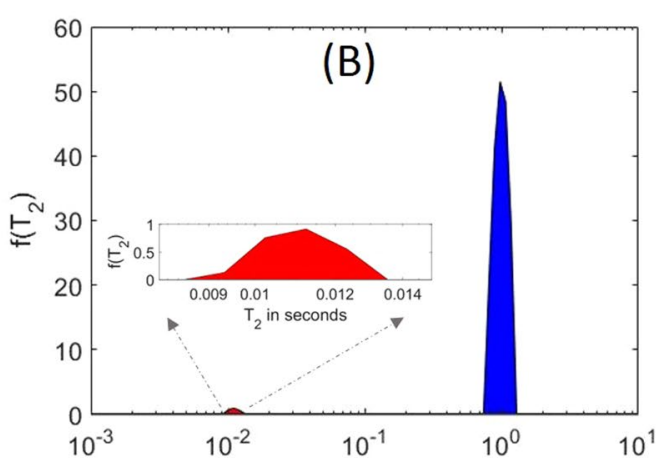

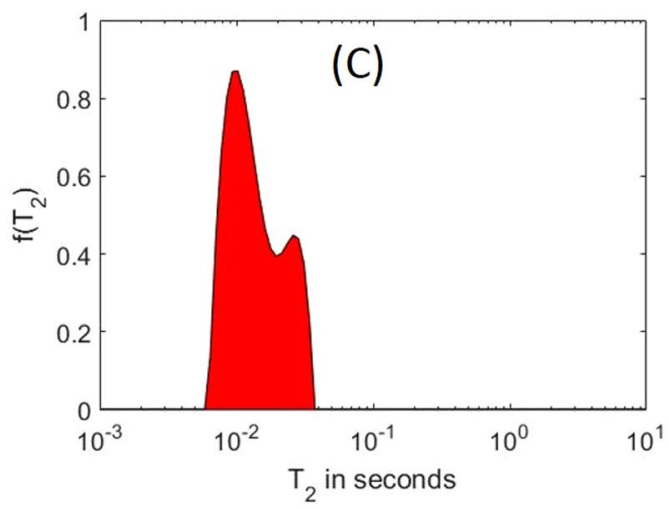

Figure 1. Characterization of the extruded PEEK capillary. (A) A photo of the as-received extruded PEEK tube; (B) The $T_{2}$ spectrum of dodecane contained in the PEEK tube, with the inset showing the zoom-in of the fastrelaxation component; (C) The $T_{2}$ spectrum of a dried PEEK tube.

\section{Capillary Tube}

Functions of the capillary tube are two-fold: it contains the sample under study and provides the mechanical structure to which the RF antenna is attached. Accordingly, the capillary material needs to be non-magnetic, non-conductive, ideally of zero magnetic susceptibility, easy to machine, and can withstand HTHP conditions. The tube is also required to have an ultra-smooth inner wall that is in direct contact with the sample, minimizing surface-induced mobility restriction of fluid molecules.

For the HTHP sensor, we chose PEEK (polyether ether ketone) as the capillary material thanks to its high melting point $\left(335^{\circ} \mathrm{C}\right)$, relatively high tensile strength, and exceptional chemical and hydrolysis resistance. To avoid contaminating the capillary inner wall, neither physically nor chemically, we used extruded PEEK tubes by Zeus Inc, with an OD of $5.3 \mathrm{~mm}$ and an ID of $1.6 \mathrm{~mm}$, as the raw material shown in Fig. 1A.

To evaluate contributions to the measured signal from protons in the capillary material, we wound a solenoidal coil of $1 \mathrm{~cm}$ in length over the PEEK tube. We then placed the dodecane-filled capillary tube inside a Halbach-array magnet of $0.54 \mathrm{~T} B_{0}$ and performed NMR CPMG measurements ${ }^{34,35}$ with a Kea2 spectrometer by Magritek. Subsequently, a fast Laplace inversion (FLI) routine ${ }^{36}$ was applied to invert time-domain signals to distributions of $T_{2}$ relaxation times.

As shown in Fig. 1B, a sharp peak was observed at $T_{2} \sim 1 \mathrm{~s}$, corresponding to relaxation times of the bulk fluid under ambient conditions $\left(\mathrm{T}=21^{\circ} \mathrm{C}, \mathrm{P}=1 \mathrm{bar}\right.$ ), accompanied by a small peak at $T_{2}=10 \mathrm{~ms}$. To clarify its genesis, we conducted a control experiment on the same but completely dried tube, as shown in Fig. 1C. Since the fast-relaxation component of equal amplitude persisted, we assigned its origin to the PEEK matrix.

NMR relaxometry is a volumetric measurement, so the ratio of integrals under two spectral peaks represents relative signal strength of their respective origins. In this case, the PEEK signal was $2 \%$ of the fluid peak. Furthermore, the modified capillary installed in the NMR probe had an OD of $3.2 \mathrm{~mm}$ in the antenna section, corresponding to a further $63.5 \%$ reduction of the polymer volume from the as-received tubes. We later show that the matrix contribution was negligible in an assembled NMR probe.

When the operating temperature rises above the PEEK's glass transition temperature, $143^{\circ} \mathrm{C}$, stress accumulated during the manufacturing process will be released, resulting in a $2 \%$ shortening of the tube length. To avoid the deformation, we annealed the polymers at $200^{\circ} \mathrm{C}$ per the procedure in Table 1 . The annealed capillaries were further machined to fit in an HTHP NMR probe.

\section{HTHP NMR Probe}

To minimize the differential pressure across the thin wall of PEEK capillaries, we designed a pressure-compensation fixture shown in Fig. 2. The idea was to immerse the capillary in a bath of fluorinated hydrocarbons (Fluorinert by $3 \mathrm{M}$ ) that didn't contain any protons. Fluorinert acted as a pressure-compensation fluid that was separated from the sample fluid by a movable piston, so that the two fluids had no substance exchange while maintaining pressure 


\begin{tabular}{|l|l|l|l|}
\hline Start temp $\left({ }^{\circ} \mathrm{C}\right)$ & End temp $\left({ }^{\circ} \mathrm{C}\right)$ & Ramp rate $\left({ }^{\circ} \mathrm{C} / \mathbf{m i n}\right)$ & Duration $(\mathrm{hrs})$ \\
\hline 20 & 150 & 0.1 & 21.7 \\
\hline 150 & 150 & 0 & 3 \\
\hline 150 & 200 & 0.1 & 8.3 \\
\hline 200 & 200 & 0 & 3 \\
\hline 200 & 20 & -0.1 & 30 \\
\hline
\end{tabular}

Table 1. PEEK annealing procedure.
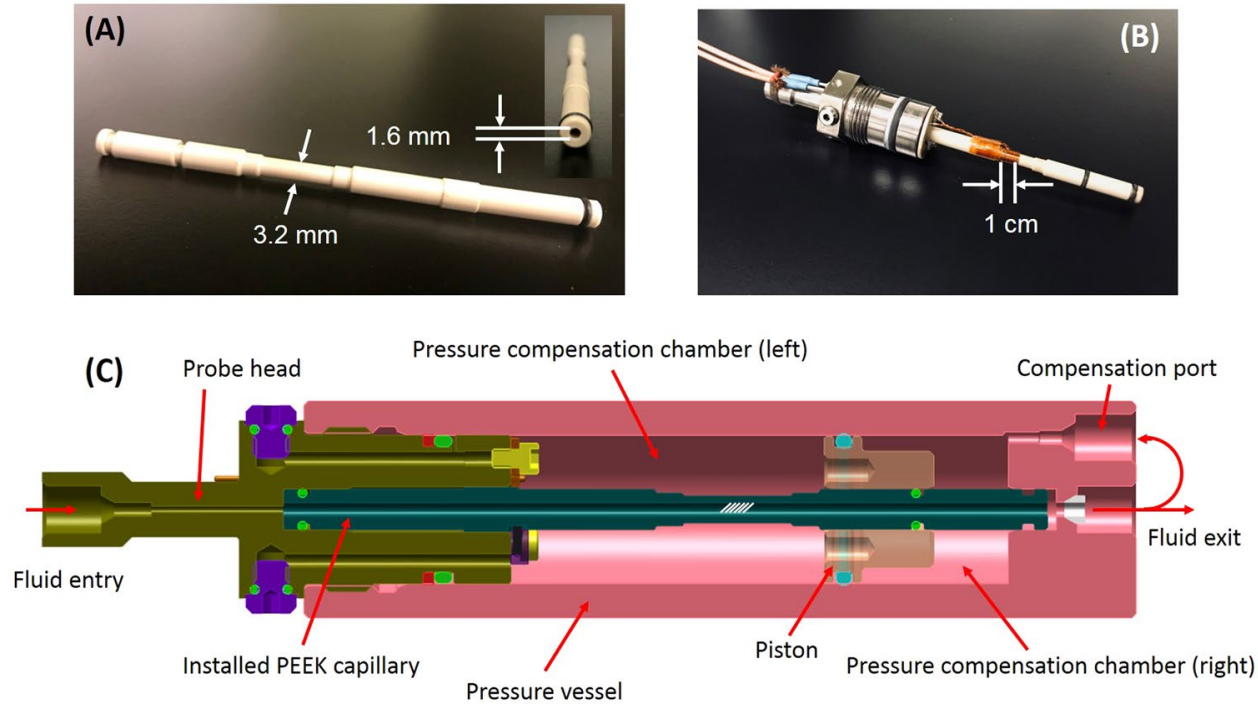

Figure 2. The HTHP NMR probe. (A) The machined PEEK capillary, both side view and front view (inset); (B) The installed PEEK capillary on the probe head; (C) A schematic cross section of the HTHP probe. To equalize pressures across the capillary wall, the space in between of the capillary outer wall and the pressure vessel is filled with fluids of equal pressure. The annular space is further partitioned into two chambers (left and right) by a PEEK piston. To avoid interference with signals from the sampled fluids, the pressure-compensation fluids in the left chamber are fluorinated hydrocarbons. In contrast, the fluids in the right chamber come from the capillary, which enter the space through the compensation port. When the operating condition varies, the piston moves so as to maintain pressure equilibrium across the capillary wall.

communication. When the operating condition varied, the piston moved to equalize hydraulic pressures across the capillary wall. All the fluids were contained in a vessel made of titanium alloy that could hold a differential pressure up to 900 bar.

To achieve pressure management while meeting the spectrometer specifications, we further machined the PEEK tube that included three O-ring grooves and indentations, as shown in Fig. 2A. During the machining process, the inner wall of the tube was supported by a through-hole gauge pin, and was therefore preserved in its pristine condition. The section of antenna placement had an OD of $3.2 \mathrm{~mm}$, an ID of $1.6 \mathrm{~mm}$, and an effective sample volume of $17 \mu \mathrm{L}$.

Subsequently, a solenoidal coil of 11 turns of 28 AWG wires, with inductance $L_{c}$ of $285 \mathrm{nH}$ and resistance $R_{c}$ of $1.35 \mathrm{ohm}$ measured at the Larmor frequency of $f_{L} \sim 23 \mathrm{MHz}$, was wound around the middle section of the machined tube. The quality factor of the coil was $2 \pi f_{L} L_{c} / R_{c}=30$. After assembling the probe, samples were introduced via the fluid entry while NMR measurements were performed within the shaded section of the capillary in Fig. 2C.

\section{Magnet, Electronics, and The System}

All the experiments were performed with a samarium-cobalt Halbach magnet, designed and manufactured by One Resonance Sensors, LLC. The magnet had a construct of hollow cylinder, with an ID of $25.4 \mathrm{~mm}$, an OD of $70 \mathrm{~mm}$, and a length of $76 \mathrm{~mm}$. A $17 \mu \mathrm{L}$ cylindrical measurement volume of ca. $100 \mathrm{ppm}$ field homogeneity, with a diameter of $1.5 \mathrm{~mm}$ and length of $1 \mathrm{~cm}$, was created at the center of the magnet's physical envelope.

The ${ }^{1} \mathrm{H}$ Larmor frequency, determined by the magnetic field strength, reduced from $23.42 \mathrm{MHz}$ at $21^{\circ} \mathrm{C}$ to $22.25 \mathrm{MHz}$ at $150^{\circ} \mathrm{C}$. Thanks to the low thermal coefficient of $\mathrm{SmCo}$ (below13 ppm $/{ }^{\circ} \mathrm{C}$ ) and a large thermal mass of the setup, drifts of $B_{0}$ were kept well below $100 \mathrm{~Hz}$ during any single experiment through the entire temperature range. In conjunction with the HTHP probe and the magnet, we developed a fully integrated NMR spectrometer.

The whole system is shown in Fig. 3A, where the single-board ( $50 \mathrm{~mm}$ by $250 \mathrm{~mm}$ ) electronics is interfaced to a laptop through a USB cable for both power and data communication. The NMR ASIC is the crucial component that is composed of an RF transmitter (TX), an RF receiver (RX), and an arbitrary pulse sequencer (APS) ${ }^{6}$. The 

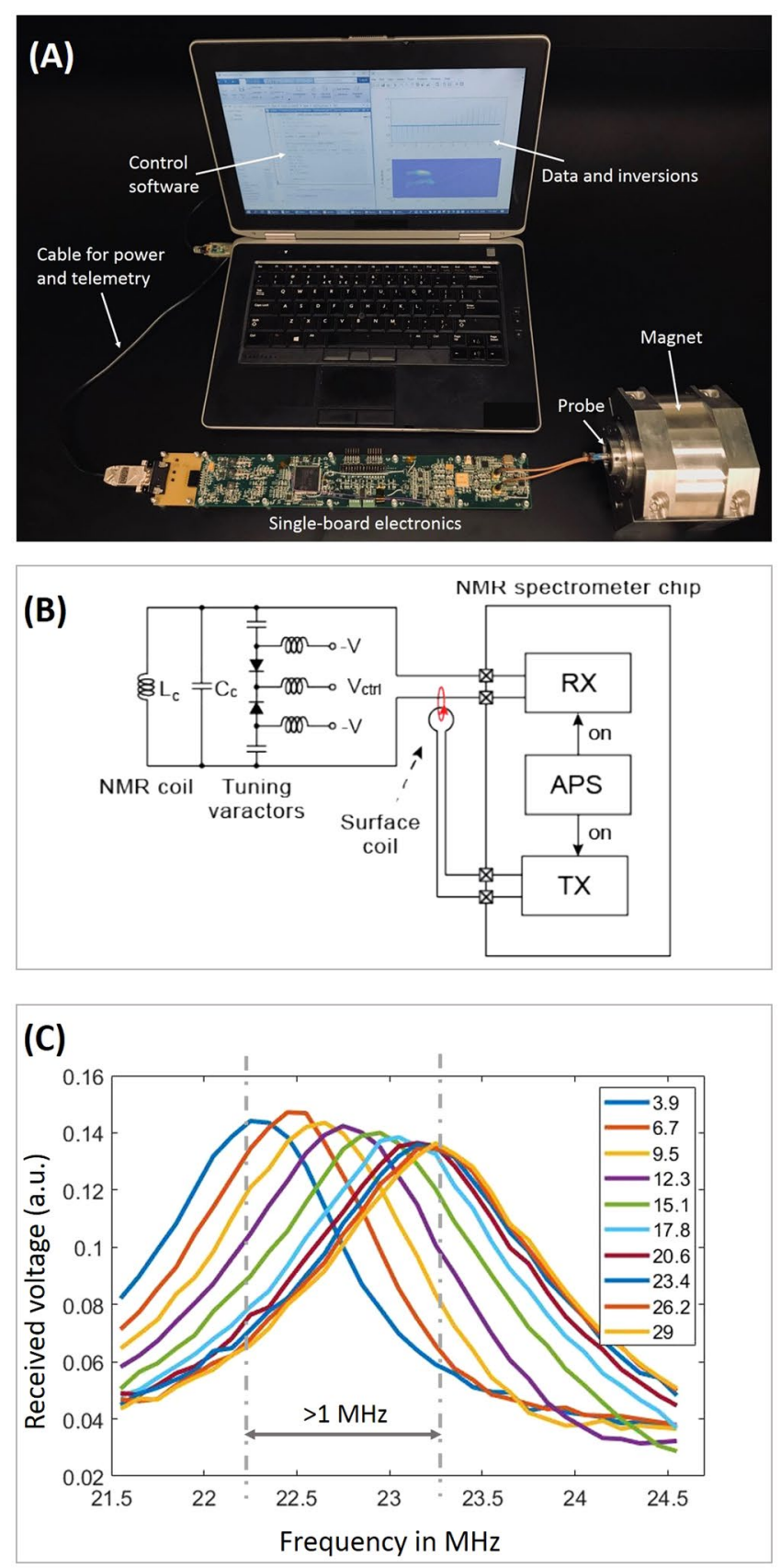

Figure 3. (A) A photo of the miniaturized NMR system that includes the magnet, the HTHP probe, the single-board electronics, and a laptop. The power is drawn from the laptop through the USB cable, which also transmits data and commands; (B) The schematic of circuit configuration with a small surface coil that injects RF energy into the NMR antenna for circuit tuning; (C) The amplitude of received signal at the on-chip receiver as a function of sweeping frequency for different reverse-biased voltage. This experiment was carried out under ambient conditions.

chipset executes pulse programs by delivering RF pulse trains to and acquiring signals from the NMR antenna; a frequency synthesizer, including a PLL (HMC832 by Analog Device) and a quartz oscillator (PX570 by Vectron), provides the radiofrequency reference; and a microcontroller (TMS320F28335PTPQ by Texas Instruments) synthesizes the pulse programs, preprocesses acquired data, and relays the data to the laptop.

Other components include a DAC and a varactor network for circuit tuning, two switches for RF isolation, ADC for signal digitization, power management ICs, and temperature sensors. The spectrometer, with a total power budget of $2 \mathrm{~W}$, can deliver up to $300 \mathrm{~mW}$ in RF transmission. More information of the board architecture and operating principles may be found in the Supplementary Materials.

When temperature drifts widely, it is important to match circuit resonance to the proton Larmor frequency, $f_{L}$. Circuit tuning is achieved by varying capacitance of the varactor arrays (BB639C by Infineon Technologies) in 
(A)

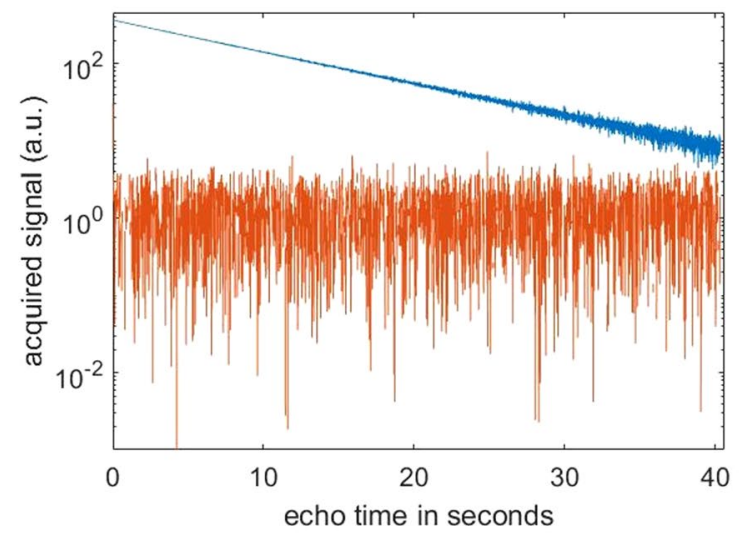

(B)
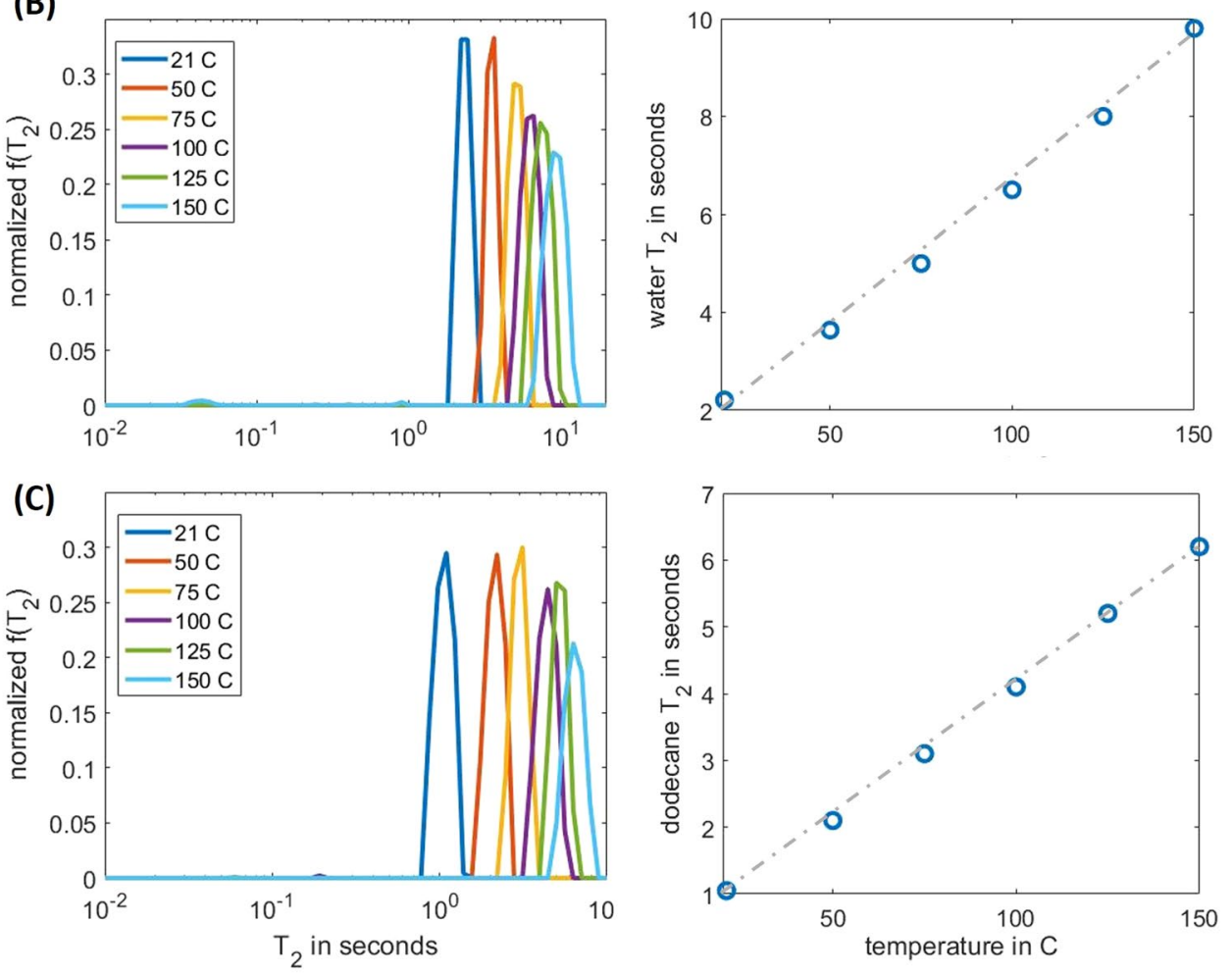

Figure 4. HTHP NMR relaxometry of bulk fluids at different temperatures. (A) In-phase (blue) and out-ofphase (red) time-domain relaxation data for water at $150{ }^{\circ} \mathrm{C}$ and $137 \mathrm{bar}$; (B) (left) Normalized $T_{2}$ spectra of water at different temperatures, and (right) the corresponding log mean; (C) (left) Normalized $T_{2}$ spectra of dodecane at different temperatures, and (right) the corresponding log mean.

Fig. 3B, where resonance frequency is given by $f_{c}=1 /\left(2 \pi \sqrt{\left(C_{c}+C_{v}\right) L_{c}}\right)$ with $L_{c}$ the coil inductance, $C_{c}=160 \mathrm{pF}$ a fixed tuning capacitor, and $C_{v}$ the varactor capacitance.

During circuit tuning, a small surface coil injects a fraction of transmitted energy into the NMR antenna, and the amplified signal is detected by the on-chip receiver. By monitoring the signal amplitude, we could determine conditions of circuit resonance. As shown in Fig. 3C, we performed an experiment under ambient conditions on recording the antenna response as a function of sweeping input frequency. As the applied reverse bias increased from $3.9 \mathrm{~V}$ to $29 \mathrm{~V}, f_{c}$ shifted by more than $1 \mathrm{MHz}$. In practice, the circuit response needs to be characterized and tabulated for realtime adjustments.

\section{HTHP NMR Relaxometry}

To characterize the HTHP probe, we conducted NMR relaxation measurements with a Kea2 spectrometer by Magritek. The probe, together with the SmCo magnet, was placed in a temperature-controlled oven. High-pressure fluid lines were connected to the probe for feeding in samples from a micro-reactor (PN 212340E by HiP Company), while hydraulic pressures were regulated by a syringe pump ( $\mathrm{D}$-series by Teledyne).

We ran CPMG experiments on two samples, DI water and dodecane, from $21^{\circ} \mathrm{C}$ to $150^{\circ} \mathrm{C}$ at a constant hydraulic pressure of 137 bar. To measure long $T_{2}$ 's, it is essential to maintain spin coherence over an extended 

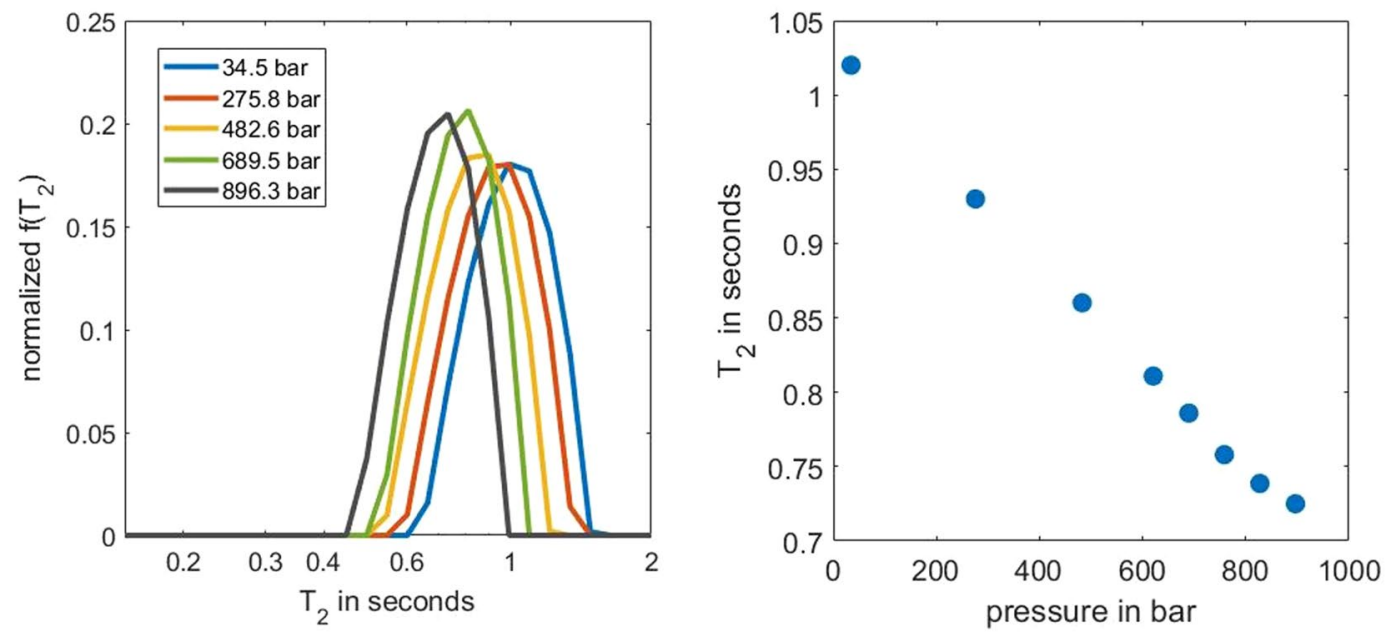

Figure 5. (left) Five selected $T_{2}$ spectra of dodecane at $28^{\circ} \mathrm{C}$ with varying hydraulic pressures from 34 bar to 896 bar; (right) The log mean of the $T_{2}$ spectra as a function of pressure.

duration, as shown in Fig. 4A. The Kea2 spectrometer used an oven-controlled oscillator with a \pm 1 ppb stability. At $23 \mathrm{MHz}$ operating frequency, such stability allows to reliably maintain phase coherence in a CPMG train up to $45 \mathrm{~s}$ echo time. Consequently, the system is capable of measuring $T_{2}$ up to ca. $10 \mathrm{~s}$.

For both fluids, the temperature-dependent $T_{2}$ spectra were clean and narrowly distributed (Fig. 4B,C), consistent with the characteristics of pure bulk fluids. The obtained $T_{2}$ at $21^{\circ} \mathrm{C}$ were consistent with measurements from samples of much larger volume $(10 \mathrm{~mL})$ measured at a commercial spectrometer (RCA by Magritek) with a substantially smaller $B_{0}$ gradient, signifying minimal effects of $B_{0}$ inhomogeneity in our system. In addition, both samples showed a linear dependence of $T_{2}$ over temperature, a hallmark of motion-averaging regime ${ }^{37}$. Specifically, the slope of measured water $T_{2}$ agreed with published results in $^{26}$, while the effect of dissolved oxygen $^{38}$ barred a direct comparison ${ }^{39}$ on dodecane. We also note that the spectra appeared somewhat broadened at elevated temperatures, which might be attributed to deteriorated system SNRs.

We further tested the system by varying hydraulic pressure from 34 bar to 896 bar on a dodecane sample at $28^{\circ} \mathrm{C}$, which resulted in a monotonic diminishing $T_{2}$ up to $28 \%$, as shown in Fig. 5 . As pressure increased, the more densely packed molecules suppressed their relative motion, and consequently $T_{2}$ 's decreased ${ }^{40}$. The result agreed with previously reported results ${ }^{39}$, assuming a constant oxygen effect (Supplementary Materials).

\section{The Miniaturized Spectrometer}

Beyond the pure compounds, we benchmarked the fully integrated NMR platform on a suite of complex fluids, both in their chemical composition and spatial configuration, that include food ingredients and multiphasic hydrocarbons. To resolve multiple chemical species and their interactions, we programmed the system to perform 2D $T_{1}-T_{2}$ correlation spectroscopy ${ }^{41}$.

As shown in Fig. 3A, a pulse program is initiated in Matlab at the laptop with a set of pulsing and acquisition parameters, which is transmitted to the microprocessor and further relayed to the NMR ASIC for execution. For acquiring a $T_{1}-T_{2}$ spectrum, we used the inversion recovery-CPMG (IRCPMG) sequence with the function ${ }^{41}$ :

$$
S\left(\tau_{1}, \tau_{2}\right)=\int\left(1-2 e^{-\tau_{1} / T_{1}}\right) e^{-\tau_{2} / T_{2}} f\left(T_{1}, T_{2}\right) d T_{1} d T_{2},
$$

where $\tau_{1}$ and $\tau_{2}$ are $T_{1}$ encoding and echo time, respectively, with corresponding signals $S\left(\tau_{1}, \tau_{2}\right)$ shown in Fig. 6A. Subsequently, the FLI routine is applied that inverts the time-domain signal to a $T_{1}-T_{2}$ correlation distribution, $f\left(T_{1}, T_{2}\right)$.

We show $T_{1}-T_{2}$ spectra of three fluids with measurements taken at echo spacing $=0.25 \mathrm{~ms}$. Under ambient conditions, the system had a Gaussian noise and a single-scan SNR of ca. 40. In Fig. 6B, the water sample shows a tight peak at $T_{1}=T_{2}=2.2 \mathrm{~s}$, manifesting the unrestricted motion of molecules in their bulk state. In Fig. $6 \mathrm{C}$, a fresh egg yolk shows a spread of distribution along the line of $T_{1} / T_{2}=2$, reflecting the complex interactions of water, free-floating proteins, and protein-fat aggregates ${ }^{42,43}$. The further elevated $T_{1} / T_{2}$ ratio of the fast-relaxation components at $T_{2} \sim 4 \mathrm{~ms}$ indicates additional motion slowdowns of large molecules ${ }^{44}$.

In Fig. 6D, we performed experiments on a sample of emulsified heavy oil (with density of $0.978 \mathrm{~g} / \mathrm{cm}^{3}$ ) with water of $60 \%$ volume fraction, a common encounter in the upstream oilfield as well as refinery facilities. The turbid fluid manifested a distinctive oil peak at $T_{2} \sim 1 \mathrm{~ms}$ and a large $T_{1} / T_{2}$ ratio over 100, likely originating from a combination of slow molecular motion and proton-electron interactions by paramagnetic species in asphaltene molecules ${ }^{45}$. Comparing to bulk water, the emulsified water presented a shortened $T_{2}$ and a slightly elevated $T_{1} / T_{2}$ ratio, thanks to its interaction with the crude and emulsifiers. 
(A)

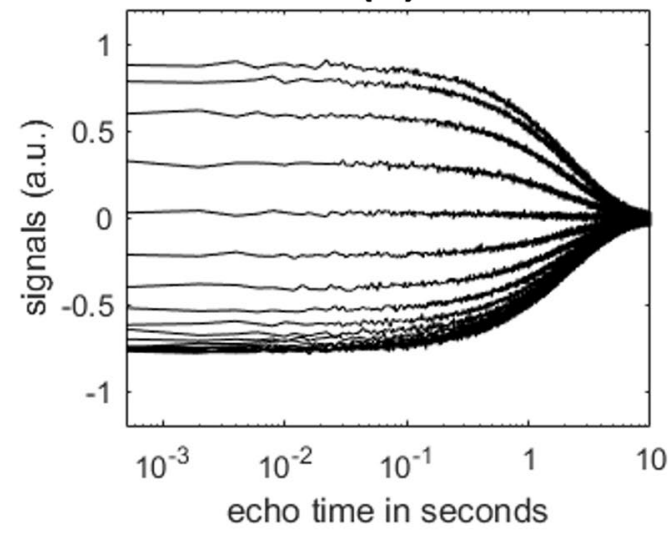

(C)

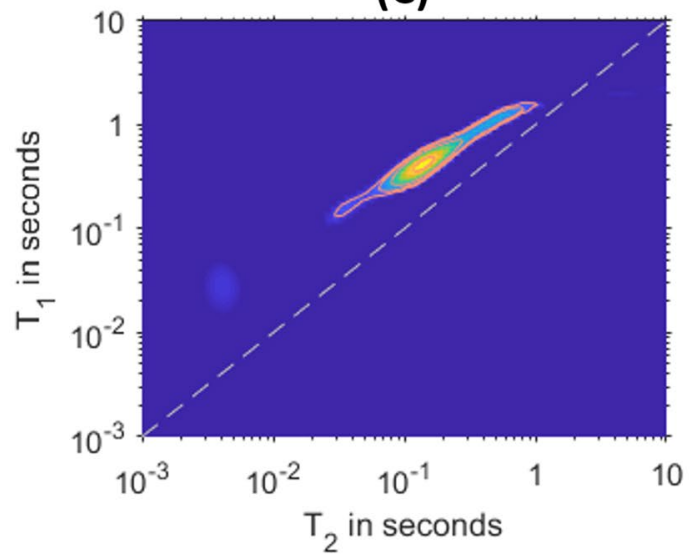

(B)

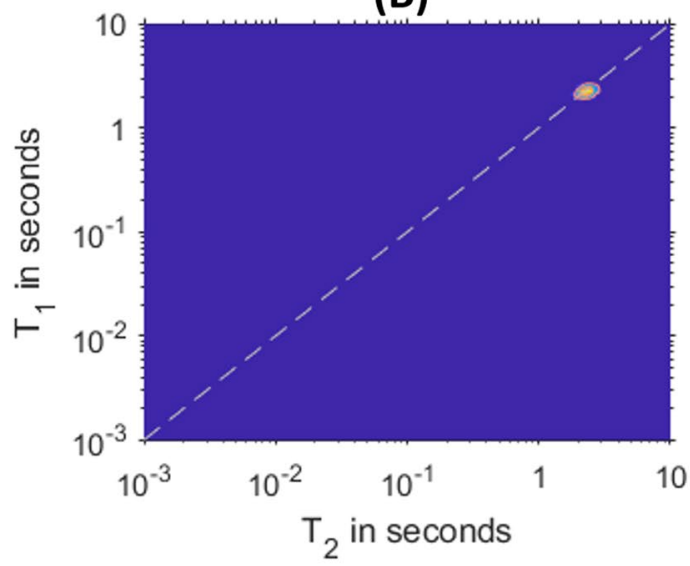

(D)

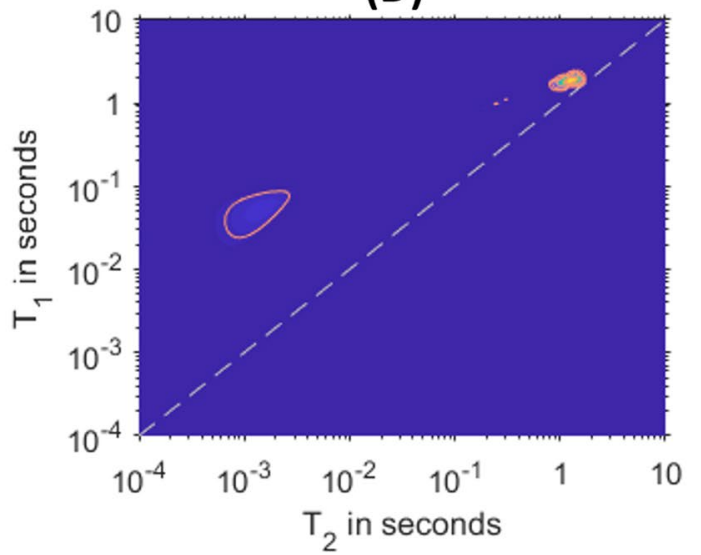

Figure 6. IRCPMG experiments performed by the miniaturized NMR spectrometer on several samples. (A) The time-domain signals of a water sample. Each trace is a CPMG train, acquired at different $\tau_{1}$ times. $T_{1}-T_{2}$ spectra for water are shown in (B), for an egg yolk in (C) and for emulsified crude oil with water in (D).

\section{Discussion}

Traditional HTHP NMR is notoriously difficult to perform, given the size and complexity of the HTHP management system. In this regard, reducing the overall sensor footprint helps tremendously alleviate the hardware requirements. Nevertheless, difficulties need to be overcome to achieve the designed functionalities, such as elimination of surface effects, pressure compensation of the probe, and tight control of temperature drift and of overall system stability, including electronics, phase coherence, and sample configurations.

In this work, geometry of the NMR probe is optimized for minimizing frictions in introducing and discharging samples. This "flowline" design is ideal for applications where frequent fluid displacements are either desired or required, such as in online monitoring of fluid properties ${ }^{15}$, investigation of chemical reactions with changing reagents ${ }^{46}$, and evaluation of sampled fluids in oil wells ${ }^{47}$.

Although we focus on relaxometry, the sensor assembly can be extended to other types of NMR measurements with relative ease. For example, a pair of small gradient coils may be added for diffusion measurements ${ }^{48}$; shimming coils and electronics may also be included for chemical-shift spectroscopy ${ }^{6}$. Those modalities can be developed separately to further expand the use of portable instruments for HPHT NMR in a wider context.

We also note that PEEK polymer has its limits. For example, it has a glass transition temperature of $143^{\circ} \mathrm{C}$ and therefore could fail at temperatures well above $T_{g}$; It is relatively weak and requires pressure compensation for HP applications, which complicates the probe design. Alternative materials, such as sapphire ${ }^{49}$, irconia $^{50}$, and diamond ${ }^{51}$, do exist and merit considerations.

\section{References}

1. Fu, R. et al. Ultra-wide bore $900 \mathrm{MHz}$ high-resolution NMR at the national high magnetic field laboratory. Journal of Magnetic Resonance 177, 1-8 (2005).

2. Hayes, C. E., Edelstein, W. A., Schenck, J. F., Mueller, O. M. \& Eash, M. An efficient, highly homogeneous radiofrequency coil for whole-body NMR imaging at 1.5 T. Journal of Magnetic Resonance 63, 622-628 (1985).

3. Kovacs, H., Moskau, D. \& Spraul, M. Cryogenically cooled probesa leap in NMR technology. Progress in Nuclear Magnetic Resonance Spectroscopy 46, 131-155 (2005).

4. Raich, H. \& Blümler, P. Design and construction of a dipolar Halbach array with a homogeneous field from identical bar magnets: NMR mandhalas. Concepts in Magnetic Resonance Part B: Magnetic Resonance Engineering: An Educational Journal 23, 16-25 (2004). 
5. Danieli, E., Perlo, J., Blümich, B. \& Casanova, F. Small magnets for portable NMR spectrometers. Angew. Chem. Int. Ed 49, 4133-4135 (2010).

6. Ha, D., Paulsen, J. L., Sun, N., Song, Y.-Q. \& Ham, D. Scalable NMR spectroscopy with semiconductor chips. Proc. Natl. Acad. Sci. USA 111, 11955-11960 (2014).

7. Huber, S. et al. Multichannel digital heteronuclear magnetic resonance biosensor. Biosensors and Bioelectronics 126, 240-248 (2019).

8. Renaud, L. et al. Implantable planar rf microcoils for NMR microspectroscopy. Sensors and Actuators A: Physical 99, 244-248 (2002).

9. Wensink, H. et al. Measuring reaction kinetics in a lab-on-a-chip by microcoil NMR. Lab on a Chip 5, 280-284 (2005).

10. Zalesskiy, S. S., Danieli, E., Blumich, B. \& Ananikov, V. P. Miniaturization of NMR systems: Desktop spectrometers, microcoil spectroscopy, and NMR on a chip for chemistry, biochemistry, and industry. Chemical Reviews 114, 5641-5694 (2014).

11. Lee, H., Sun, E., Ham, D. \& Weissleder, R. Chip-NMR biosensor for detection and molecular analysis of cells. Nature Medicine 14, 869 (2008).

12. Jeong, S. et al. Real-time quantitative analysis of metabolic flux in live cells using a hyperpolarized micromagnetic resonance spectrometer. Science Advances 3, e1700341 (2017).

13. Fridjonsson, E. O., Stanwix, P. L. \& Johns, M. L. Earths field NMR flow meter: Preliminary quantitative measurements. Journal of Magnetic Resonance 245, 110-115 (2014).

14. Osán, T. et al. Fast measurements of average flow velocity by Low-Field ${ }^{1} \mathrm{H}$ NMR. Journal of Magnetic Resonance 209, 116-122 (2011).

15. Kumar, S. Liquid-contents verification for explosives, other hazards, and contraband by magnetic resonance. Applied Magnetic Resonance 25, 585 (2004).

16. Pinter, M., Harter, T., McCarthy, M. \& Augustine, M. Towards using NMR to screen for spoiled tomatoes stored in 1,000 L, aseptically sealed, metal-lined totes. Sensors 14, 4167-4176 (2014).

17. Chen, J. J. et al. Ex situ nmr relaxometry of metal-organic frameworks for rapid surface-area screening. Angew. Chem. Int. Ed 52, 12043-12046 (2013).

18. Fantazzini, P. et al. Gains and losses of coral skeletal porosity changes with ocean acidification acclimation. Nature Communications 6, 7785 (2015).

19. Blumich, B. et al. Noninvasive testing of art and cultural heritage by mobile NMR. Accounts of Chemical Research 43, 761-770 (2010).

20. Del Federico, E. et al. Unilateral NMR applied to the conservation of works of art. Analytical and Bioanalytical Chemistry 396, 213-220 (2010).

21. Eidmann, G., Savelsberg, R., Blümler, P. \& Blümich, B. The nmr mouse, a mobile universal surface explorer. Journal of Magnetic Resonance 122, 104-109 (1996).

22. Sun, N. et al. Palm NMR and 1-chip NMR. IEEE Journal of Solid-State Circuits 46, 342-352 (2011).

23. Bloembergen, N., Purcell, E. M. \& Pound, R. V. Relaxation effects in nuclear magnetic resonance absorption. Physical Review 73, 679 (1948).

24. Freedman, R. et al. A compact high-performance low-field NMR apparatus for measurements on fluids at very high pressures and temperatures. Rev. Sci. Instrum. 85, 025102 (2014).

25. Bertram, H. C., Kohler, A., Böcker, U., Ofstad, R. \& Andersen, H. J. Heat-induced changes in myofibrillar protein structures and myowater of two pork qualities. A combined FT-IR spectroscopy and low-field NMR relaxometry study. Journal of Agricultural and Food Chemistry 54, 1740-1746 (2006).

26. Sierra-Martin, B., Retama, J. R., Laurenti, M., Barbero, A. F. \& Cabarcos, E. L. Structure and polymer dynamics within PNIPAMbased microgel particles. Advances in Colloid and Interface Science 205, 113-123 (2014).

27. Zheng, Q. et al. Water-wax behaviour in porous silica at low temperature Fischer-Tropsch conditions. Applied Catalysis A: General 572, 142-150 (2019).

28. Baker, D. \& Conradi, M. S. Apparatus for high temperatures and intermediate pressures, for in situ nuclear magnetic resonance of hydrogen storage systems. Rev. Sci. Instrum. 76, 073906 (2005).

29. Horch, C., Schlayer, S. \& Stallmach, F. High-pressure low-field ${ }^{1} \mathrm{H}$ NMR relaxometry in nanoporous materials. Journal of Magnetic Resonance 240, 24-33 (2014).

30. Papaioannou, A. \& Kausik, R. Methane storage in nanoporous media as observed via high-field NMR relaxometry. Physical Review Applied 4, 024018 (2015).

31. Foley, I., Farooqui, S. \& Kleinberg, R. Effect of paramagnetic ions on NMR relaxation of fluids at solid surfaces. Journal of Magnetic Resonance, Series A 123, 95-104 (1996).

32. Godefroy, S., Korb, J.-P., Fleury, M. \& Bryant, R. Surface nuclear magnetic relaxation and dynamics of water and oil in macroporous media. Physical Review E 64, 021605 (2001).

33. Liu, G., Li, Y. \& Jonas, J. Confined geometry effects on reorientational dynamics of molecular liquids in porous silica glasses. The Journal of Chemical Physics 95, 6892-6901 (1991).

34. Carr, H. Y. \& Purcell, E. M. Effects of diffusion on free precession in NMR experiments. Phys. Rev. 94, 630-638 (1954).

35. Meiboom, S. \& Gill, D. Modified Spin-echo Method for Measuring Nuclear Relaxation Times. Rev. Sci. Instrum. 29, 688-691 (1958).

36. Venkataramanan, L., Song, Y.-Q. \& Hurlimann, M. D. Solving Fredholm integrals of the first kind with tensor product structure in 2 and 2.5 dimensions. IEEE Transactions on Signal Processing 50, 1017-1026 (2002)

37. Levitt, M. H. Spin dynamics: Basics of nuclear magnetic resonance (John Wiley \& Sons, 2001)

38. Mutina, A. \& Hürlimann, M. Effect of oxygen on the NMR relaxation properties of crude oils. Applied Magnetic Resonance 29, 503 (2005).

39. Zega, J. A. Spin-lattice relaxation in normal alkanes at elevated pressures. Ph.D. thesis (1991).

40. Freed, D. E. Temperature and pressure dependence of the diffusion coefficients and NMR relaxation times of mixtures of alkanes. The Journal of Physical Chemistry B 113, 4293-4302 (2009).

41. Song, Y.-Q. et al. $T_{1}-T_{2}$ correlation spectra obtained using a fast two-dimensional Laplace inversion. Journal of Magnetic Resonance 154, 261-268 (2002).

42. Laghi, L. et al. A proton NMR relaxation study of hen egg quality. Magnetic Resonance Imaging 23, 501-510 (2005).

43. McGee, H. On food and cooking: The science and lore of the kitchen (Simon and Schuster, 2007).

44. Hürlimann, M. D., Burcaw, L. \& Song, Y.-Q. Quantitative Characterization of Food Products by two-dimensional $D-T_{2}$ and $T_{1}-T_{2}$ Distribution Functions in a Static Gradient. Journal of Colloid and Interface Science 297, 303-311 (2006).

45. Zielinski, L., Saha, I., Freed, D. E., Hurlimann, M. D. \& Liu, Y. Probing asphaltene aggregation in native crude oils with low-field NMR. Langmuir 26, 5014-5021 (2010).

46. Dalitz, F., Cudaj, M., Maiwald, M. \& Guthausen, G. Process and reaction monitoring by low-field NMR spectroscopy. Progress in Nuclear Magnetic Resonance Spectroscopy 60, 52 (2012).

47. Dandekar, A. Y. Petroleum reservoir rock and fluid properties (CRC press, 2013).

48. Parker, R., Zupancic, I. \& Pirs, J. Coil system to produce orthogonal, linear magnetic field gradients. Journal of Physics E: Scientific Instruments 6, 899 (1973).

49. Arnold, M. R., Kalbitzer, H. R. \& Kremer, W. High-sensitivity sapphire cells for high pressure NMR spectroscopy on proteins. Journal of Magnetic Resonance 161, 127-131 (2003). 
50. Humphries, T. D., Birkmire, D., Hauback, B. C., McGrady, G. S. \& Jensen, C. M. In situ high pressure NMR study of the direct synthesis of $\mathrm{LiAlH}_{4}$. Journal of Materials Chemistry A 1, 2974-2977 (2013).

51. Meier, T. et al. Magnetic flux tailoring through Lenz lenses for ultrasmall samples: A new pathway to high-pressure nuclear magnetic resonance. Science Advances 3, eaao5242 (2017).

\section{Acknowledgements}

The authors would like to thank Drs Martin Hürlimann and Christopher Harrison at Schlumberger-Doll Research, and Dr. Ka-Meng Lei at Harvard University for helpful discussions; Drs Marcus Donaldson and Mark Flaum for instrument development; as well as Messrs. Bill Grant and Roman Katz for supports on the mechanical design.

\section{Author Contributions}

Y.T. and Y.S. conceived the project, Y.T. and D.M. performed the experiments, Y.T. and Y.S. wrote the paper.

\section{Additional Information}

Supplementary information accompanies this paper at https://doi.org/10.1038/s41598-019-47634-2.

Competing Interests: The authors declare no competing interests.

Publisher's note: Springer Nature remains neutral with regard to jurisdictional claims in published maps and institutional affiliations.

(c) (i) Open Access This article is licensed under a Creative Commons Attribution 4.0 International License, which permits use, sharing, adaptation, distribution and reproduction in any medium or format, as long as you give appropriate credit to the original author(s) and the source, provide a link to the Creative Commons license, and indicate if changes were made. The images or other third party material in this article are included in the article's Creative Commons license, unless indicated otherwise in a credit line to the material. If material is not included in the article's Creative Commons license and your intended use is not permitted by statutory regulation or exceeds the permitted use, you will need to obtain permission directly from the copyright holder. To view a copy of this license, visit http://creativecommons.org/licenses/by/4.0/.

(C) The Author(s) 2019 